\title{
Deployment and Utilization of Public Access Defibrillation in Taiwan
}

Jen-Tang, Sun ${ }^{\text {ba*; }}$ Yu-Chun Chiend; Yao Cheng Wangd; Yueh-Ping Liu ${ }^{b} ;$ Matthew Huei-Ming Mab,c; Wen-Chu Chiang ${ }^{\mathrm{b}, \mathrm{c}}$

a Department of Emergency Medicine, Far Eastern Memorial Hospital, New Taipei City, Taiwan, ${ }^{b}$ Department of Emergency Medicine, National Taiwan University Hospital, Taipei, Taiwan ${ }^{c}$ Department of Emergency Medicine, National Taiwan University Hospital, Yun-Lin Branch, Yun-Lin County, Taiwan, dc Fire Department, Taipei City, Taiwan

\section{Background}

Public assess defibrillation is crucial to survival of out-of-hospital cardiac arrests (OHCA). Since 2013, eight categories of public locations are required by law to install public AEDs. A compulsory National AED Registry was implemented that registered all public AEDs, and collected incident reports and electrocardiograms from OHCA with public AED use.

\section{Objective}

This study aims to review public AED installation in Taiwan since 2013, and to describe incidence and characteristics of OHCAs with public AED use.

\section{Method}

Data were collected from Taiwan Public AED Registry. Public AED installation across the country was reviewed.
Detailed characteristics of public AED uses, including incidence, locations, rescuers type, initial rhythms and BCPR(bystander-CPR), were analyzed via examining incidents reports and ECGs.

\section{Result}

From July. 2013 to Dec. 2017, 8425 public AEDs were registered and disclosed. Two leading locations of public AED installation were schools/large gathering venues $(42.17 \%)$ and others $(33.21 \%)$, while other locations showed less increase. Until Aug. 2016, 344 public AED use were reported, and 247 cases had ECGs for analysis. The top three locations with public AED use were others (34.9\%), schools/large gathering venues $(21.8 \%)$ and traffic hubs $(12.5 \%)$. Locations with highest utilization rates were longdistance vehicles $(22.4 \%)$, while schools/large gathering venues were the lowest $(2.3 \%)$. The overall shockable rhythms were $34.0 \%$; highest among schools/large gathering venues $(53.5 \%)$ and lowest among long-distance vehicles $(17.2 \%)$.
Figure 1. Distribution of Public Areas for Cases of AED used
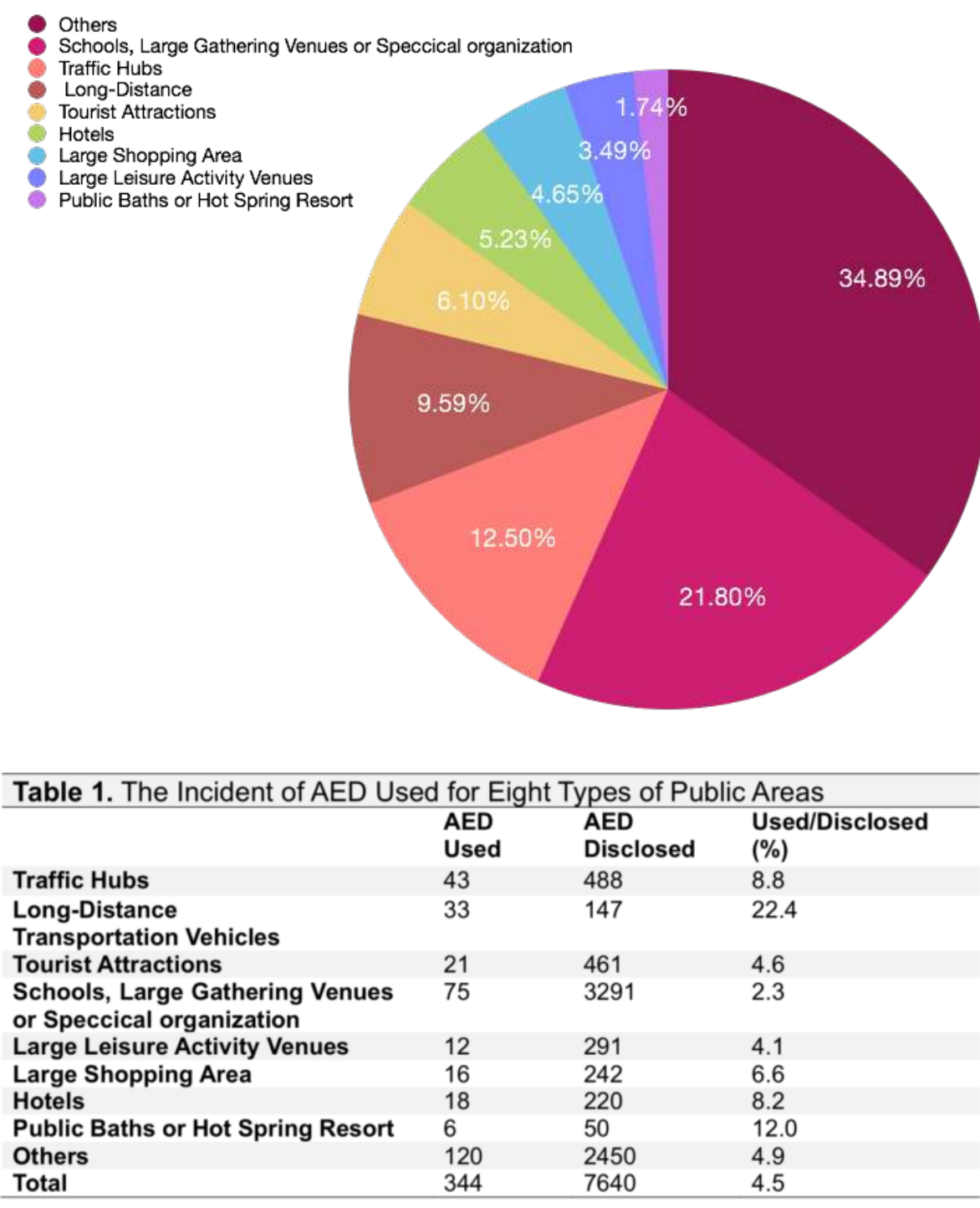

Table 2. The Rate of Shockable Rhythm among AED used for Eight Types of

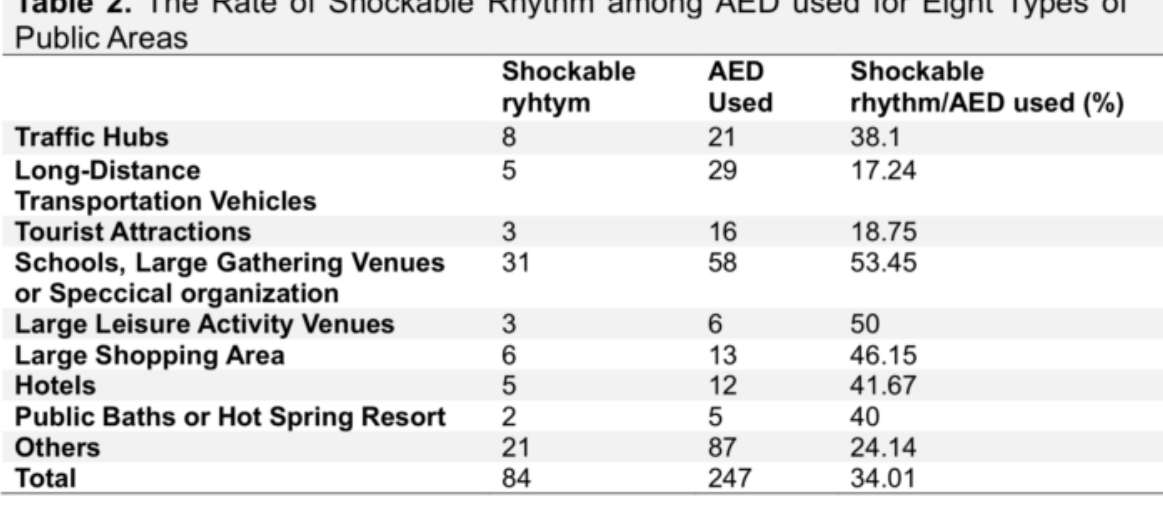

* AED used with ECG recorded
The overall ROSC rates $(\mathrm{n}=265)$ were $40.8 \%$, highest among traffic hubs(53.3\%) and lowest among large shopping areas $(25.0 \%)$. The majority of rescuers were facility employees $(\mathrm{n}=101$, $43.91 \%)$ and medical staff $(n=96$, $41.74 \%)$. Only few victim's families $(2.17 \%)$ and unrelated laypersons $(6.52 \%)$ acted as first responders. Rates of B-CPR were $69.3 \%$, and were associated with more shockable rhythm (38.4\% vs. $32.9 \%)$.

\section{Conclusion}

Public AED installed and use have rapidly increased in Taiwan since 2013. Disparities between AED installation and use among locations were observed. OHCAs with PAD use had significantly higher rates of shockable rhythms, bystander CPR, and ROSCs. Further PAD optimization should balance between installation, utilization, shockable rhythms and potential outcomes. Apart from device installations, the importance of community involvement cannot be overemphasized. 